\title{
Enhanced hard x-ray emission from microdroplet preplasma
}

\author{
M. Anand, S. Kahaly, G. Ravindra Kumar, and M. Krishnamurthy ${ }^{a)}$ \\ Tata Institute of Fundamental Research, 1 Homi Bhabha Road, Mumbai 400 005, India \\ A. S. Sandhu \\ JILA, University of Colorado, Boulder, Colorado 80303 \\ P. Gibbon \\ John-von-Neumann Institute for Computing, ZAM, Forschungszentrum Jülich, D-52425 Jülich, Germany
}

(Received 5 December 2005; accepted 22 March 2006; published online 3 May 2006)

\begin{abstract}
We perform a comparative study of hard x-ray emission from femtosecond laser plasmas in $15 \mu \mathrm{m}$ methanol microdroplets and Perspex target. The hard x-ray yield from droplet plasmas is $\simeq 68$ times more than that obtained from solid plasmas at $2 \times 10^{15} \mathrm{~W} \mathrm{~cm}^{-2}$. A $10 \mathrm{~ns}$ prepulse at about $5 \%$ of the main pulse appears to be essential for hard $\mathrm{x}$-ray generation from droplets. Hot electron temperature of $36 \mathrm{keV}$ is measured from the droplets at $8 \times 10^{14} \mathrm{~W} \mathrm{~cm}^{-2}$, whereas a three times higher intensity is needed to obtain similar hot electron temperatures from Perspex plasmas. Particle-in-cell simulations with very long scale-length density profiles support experimental observations. ( 2006 American Institute of Physics. [DOI: 10.1063/1.2200761]
\end{abstract}

The physics of laser-plasma interactions has undergone a revolution in recent times. Technological advances in lasers have opened the possibility of achieving intensities up to $10^{21} \mathrm{~W} \mathrm{~cm}^{-2}$. The nonperturbative physics of laser-matter interactions at these extreme intensities has brought forth many new concepts and applications. ${ }^{2}$ The hot dense plasma produced in such interaction has opened up novel schemes of pulsed neutron generation, ${ }^{3}$ nuclear reactions, ${ }^{4}$ table top acceleration, ${ }^{5}$ and synchrotron radiation. ${ }^{6}$ Importantly, such plasmas are promising sources of ultrashort pulse radiation in extreme ultraviolet (EUV) and x-ray regimes and increasing the efficiency of these sources is a major challenge. This obviously leads to the investigation of strategies to efficiently couple laser energy to the plasma. One such strategy has been the introduction of novel targets. Metallic nanoparticle-coated solids ${ }^{7}$ and "velvet" $\operatorname{targets}^{8}$ have yielded enhanced $\mathrm{x}$-ray emission in the moderate to very hard x-ray regime. Gaseous clusters, which are nanoparticles with solidlike local density, have been shown to absorb $90 \%$ of the incident laser energy. ${ }^{9}$ Particle acceleration up to a $\mathrm{MeV}$ and efficient nuclear fusion at intensities as low as $10^{16} \mathrm{~W} \mathrm{~cm}^{-2}$ have been observed from such cluster plasmas.

There are, however, some disadvantages in the use of gaseous clusters. A major one is the rather stringent limitation on the type of atomic or molecular species which can produce large clusters. For example, there is no simple way to generate large clusters with high- $Z$ atoms such as Pt. Besides, there is very little hard x-ray emission above $5 \mathrm{keV}$ from clusters. ${ }^{10}$ In view of these limitations, we consider liquid droplets to be a promising alternative. They are relatively debrisless and combine the advantage of size confinement with the relative ease with which a droplet can be used to introduce any atomic/molecular system of interest. ${ }^{11}$ Droplet targets have found application in EUV lithography and $\mathrm{x}$-ray microscopy. ${ }^{12,13}$ The emphasis on droplet plasma studies has so far concentrated on optimizing the EUV radiation at $13 \mathrm{~nm}$ for lithography applications, though there have been some initial studies on hard x-ray radiation. ${ }^{14-16}$ The

\footnotetext{
${ }^{a)}$ Electronic mail: mkrism@tifr.res.in
}

study of very hard x-ray emission from droplets is certainly of major interest.

In this letter, we present measurements of hard $\mathrm{x}$-ray emission (10-350 keV) from $15 \mu \mathrm{m}$ methanol droplets irradiated with $100 \mathrm{fs}$ laser pulses with intensities up to 2.2 $\times 10^{15} \mathrm{~W} \mathrm{~cm}^{-2}$. We find that a prepulse that arrives at about $10 \mathrm{~ns}$ ahead of the main pulse is critically important to generate hard $\mathrm{x}$ rays from liquid droplets at these intensities. For comparison, we measure hard x-ray emission from a solid plastic target (which has a similar atomic composition) under similar conditions. The hard $\mathrm{x}$-ray yield at $2 \times 10^{15} \mathrm{~W} \mathrm{~cm}^{-2}$ is about 68 times larger than that from the plastic under similar conditions. We also perform one dimensional (1D) particle-in-cell (PIC) simulations that help us to explain the experimental observation of enhanced x-ray generation in microdroplets.

The apparatus used in these experiments has been described elsewhere, ${ }^{17}$ and here we present only the salient features. The microdroplet targets are generated by forcing methanol through a $10 \mu \mathrm{m}$ capillary, which is modulated at $1 \mathrm{MHz}$ using a piezocrystal. The uniformly sized droplets were characterized by imaging of the droplet, and also by observing morphological dependent resonances (MDRs). ${ }^{18}$ The inset of Fig. 1(a) shows the droplets along with the image of a $25 \mu \mathrm{m}$ slit used for calibration. The droplets are produced inside a vacuum chamber maintained at $10^{-5}$ Torr. We focus the $100 \mathrm{fs}$ pulses of $800 \mathrm{~nm}$ light using a $30 \mathrm{~cm}$ planoconvex lens and achieve intensities up to 2 $\times 10^{15} \mathrm{~W} \mathrm{~cm}^{-2}$. A two-pulse setup is used to obtain a prepulse at about $10 \mathrm{~ns}$ ahead of the main pulse and a pair of polarizers together with a half-wave plate is used to control its intensity. Comparative experiments with solids are performed on an optically flat Perspex by focusing $p$-polarized light at $45^{\circ}$ incident angle with a $20 \mathrm{~cm}$ lens to a spot size of $20 \mu \mathrm{m}$ and achieve intensities up to $5 \times 10^{15} \mathrm{~W} \mathrm{~cm}^{-2}$. We used plastic Perspex targets for comparison since their atomic composition is close to that of methanol. The target is scanned such that each laser pulse is incident at a fresh portion of the target. ${ }^{7}$ The x-ray detector in all experiments is a $\mathrm{NaI}(\mathrm{Tl})$ detector, appropriately time gated with the laser 

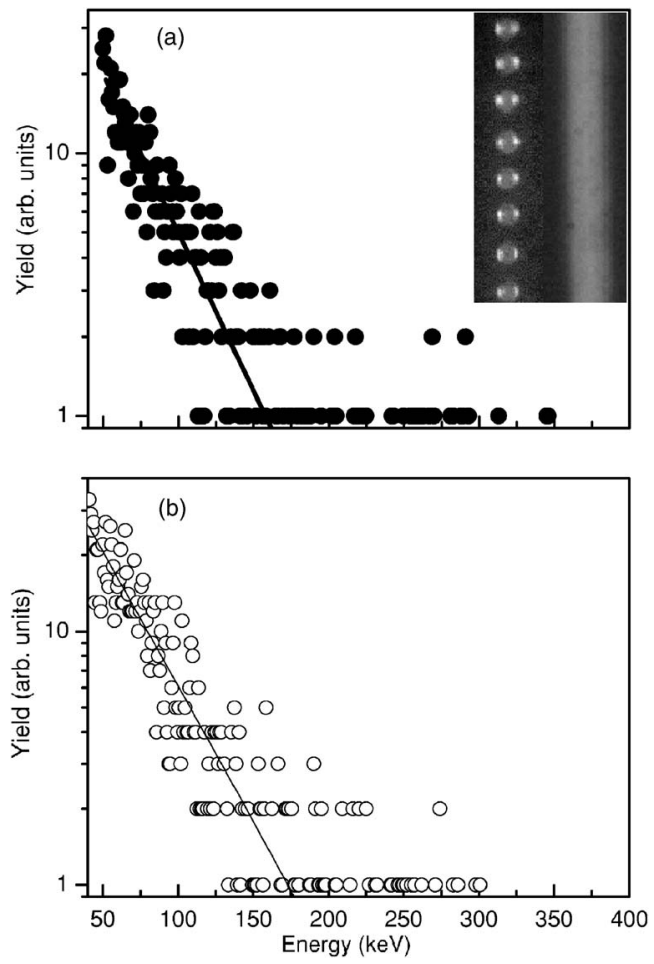

FIG. 1. X-ray emission spectra obtained when $15 \mu \mathrm{m}$ methanol droplet is irradiated at $8 \times 10^{14} \mathrm{~W} \mathrm{~cm}^{-2}$. The inset shows an image of the droplet stream along with the image of a precision $25 \mu \mathrm{m}$ slit used for determining the size of the droplet. (b) X-ray emission spectra obtained when a solid plastic target is irradiated with similar laser pulses at an intensity of 2 $\times 10^{15} \mathrm{~W} \mathrm{~cm}^{-2}$ at $45^{\circ}$ to the normal using $p$-polarized light. The solid lines show the least square fits assuming a Maxwellian distribution for electron of temperatures of $36 \mathrm{keV}$ [for droplets in (a)] and $40 \mathrm{keV}$ [for solid target in (b)].

pulse and calibrated with standard radioactive sources. The detector was placed about $50 \mathrm{~cm}$ away from the plasma source.

In experiments with liquid droplets, there is negligible hard $\mathrm{x}$-ray emission at intensities $<3 \times 10^{15} \mathrm{~W} \mathrm{~cm}^{-2}$ in the absence of a prepulse. In the regenerative amplifier, a prepulse can be generated by misalignment of the pockel cell. In initial experiments, we found that the hard x-ray generation was very sensitive to the extent of this prepulse, which is $10 \mathrm{~ns}$ ahead of the main pulse. Once we established that a nanosecond prepulse is essential for the hard $\mathrm{x}$-ray generation from the droplet, we set up a two-pulse experiment to introduce a deliberate prepulse of controllable intensity arriving at the required time ahead of the main pulse. We find that while a prepulse that is $1-10 \mathrm{ps}$ ahead does not significantly influence the $\mathrm{x}$-ray emission from the droplets, a prepulse that is about $10 \mathrm{~ns}$ ahead is essential to produce $\mathrm{x}$ rays from the droplets. The x-ray yield increases steeply with the prepulse energy and saturates for a prepulse that is about $5 \%$ in intensity of the main pulse at $1.2 \times 10^{15} \mathrm{~W} \mathrm{~cm}^{-2}$. The inset of Fig. 2 shows the change in x-ray yield measured as the fraction of prepulse energy increases up to $28 \%$ of the main pulse energy.

The x-ray emission spectrum obtained from $15 \mu \mathrm{m}$ methanol droplets at a prepulse intensity of about $5 \%$ is shown in Fig. 1(a). The data were collected for 10000 shots. The incident laser intensity was about $8 \times 10^{14} \mathrm{~W} \mathrm{~cm}^{-2}$. The solid line shows an exponential fit to the data assuming a Maxwellian distribution for the electrons in the plasma. In this fit we only considered energies larger than $50 \mathrm{keV}$ so Downloaded 21 Dec 2006 to 134.94.122.39. Redistribution subject

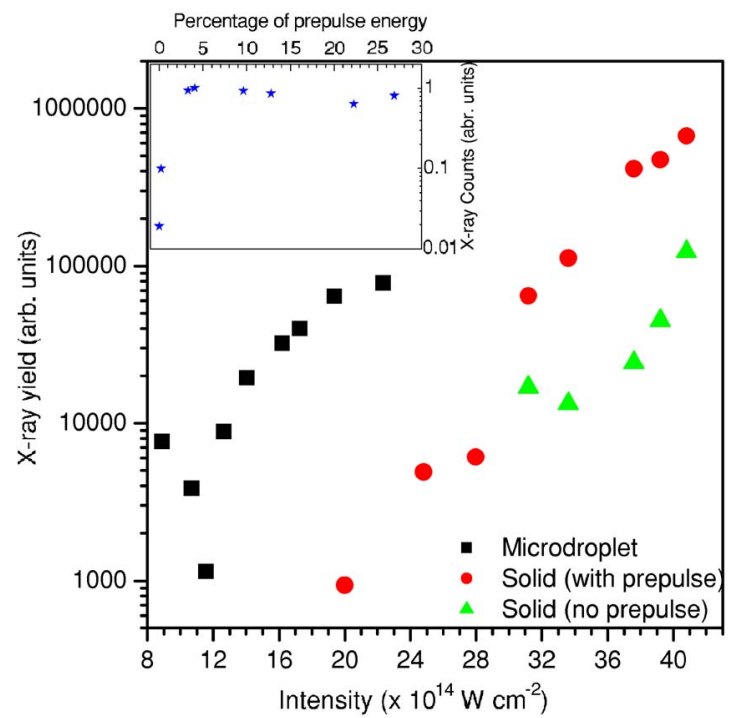

FIG. 2. (Color online) X-ray emission yields measured for $15 \mu \mathrm{m}$ methanol liquid droplet targets (squares) and solid plastic target (circles) as a function of the incident intensities and pulse energies. The x-ray yield from solid target without prepulse is also shown (triangles).

that corrections due to the transmission through the glass or aluminum housing of the detector are negligible. To avoid pileup, the count rate was kept less than 0.1 per pulse by restricting the solid angle of detection. ${ }^{17}$ The $\mathrm{x}$-ray emission spectrum from the plastic at similar prepulse intensities is shown in Fig. 1(b) at about three times larger main pulse intensity, as there was no measurable $\mathrm{x}$-ray emission below $2 \times 10^{15} \mathrm{~W} \mathrm{~cm}^{-2}$. Exponential fits to the data show that the hot electron temperature is about $40 \mathrm{keV}$ for plastic while it is $36 \mathrm{keV}$ in the case of methanol droplets at about three times less intensity. The electron temperature was measured at different intensities for the methanol droplet and was found to change from 20 to $50 \mathrm{keV}$ within the range of intensities $\left[(8-22) \times 10^{14} \mathrm{~W} \mathrm{~cm}^{-2}\right]$ accessed in our experiments. We, however, elaborate only on the x-ray yield measurements in this letter.

A comparison between the relative integrated $\mathrm{x}$-ray yields from both droplet plasma and plastic target for various incident intensities, with a prepulse of about 1.5 $\times 10^{14} \mathrm{~W} \mathrm{~cm}^{-2}$, is shown in Fig. 2. The total $\mathrm{x}$-ray yields from both the targets are measured in the range from 10 to $350 \mathrm{keV}$. Experiments on liquid drops with higher intensities are not possible with our present laser, as we are constrained to maintain a focal spot size of $30 \mu \mathrm{m}$ to maintain the droplet close to the center of the focus, given the spatial jitter of a few microns in the jet. Though the prepulse brings about 17-fold enhancement in the x-ray yield at 3.7 $\times 10^{15} \mathrm{~W} \mathrm{~cm}^{-2}$ from the plastic Perspex target, the total $\mathrm{x}$-ray yield from the methanol droplet is much larger in the presence of the prepulse. The threshold for hard x-ray generation in droplets is a factor of 2 smaller, and at an intensity of about $2 \times 10^{15} \mathrm{~W} \mathrm{~cm}^{-2}$, the $\mathrm{x}$-ray yield from droplets is at least 68 times larger than that obtained from the plastic.

In plasmas made of mesoscopic matter, both the geometry and the size are crucially important. A microdroplet is a spherical cavity that can focus the light inside the drop. A major fraction of the prepulse $\left(10^{13} \mathrm{~W} \mathrm{~cm}^{-2}\right)$ that enters the droplet can be focused, enhancing the intensity by more than two orders of magnitude. ${ }^{19}$ For our droplet size, Lorentz-Mie calculations $\mathrm{s}^{17}$ show that a maximum intensity enhancement o AlP license or copyright, see http://apl.aip.org/apl/copyright.jsp 


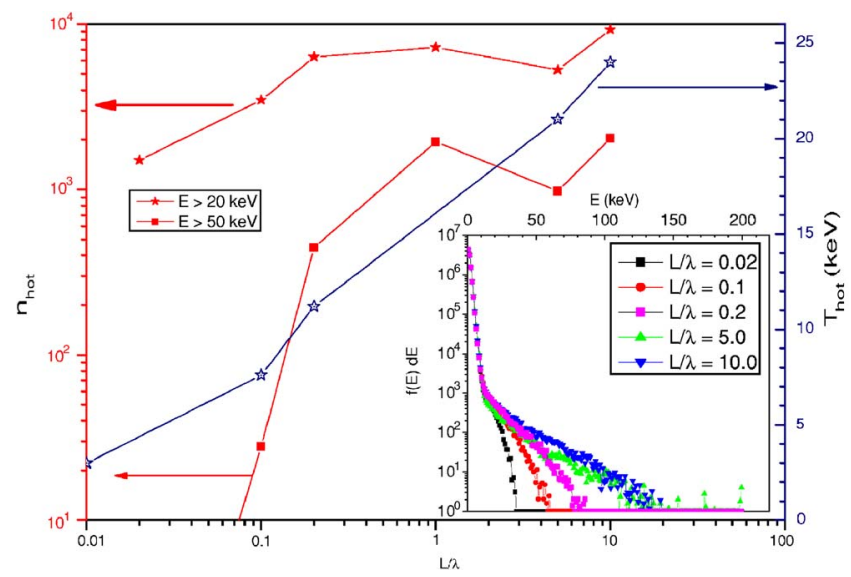

FIG. 3. (Color online) Hot electron numbers extracted from spectra as a function of density gradient. The lines are drawn to guide the eye. Inset: hot electron spectra for scale lengths $L / \lambda=0$ and $L / \lambda=10$. The inset shows the hot electron energy distribution for different $L / \lambda$.

of nearly 150 times the incident light intensity is possible inside the droplet.

Focusing of the prepulse in a liquid drop results in substantial ionization at many spots in and around the drop ${ }^{19}$ and leads to a large volume spherical plasma. Imaging experiments using the pump-probe technique show that the droplet plasma is of $30 \mu \mathrm{m}$ in diameter when the main pulse is incident. ${ }^{17}$ In this case, therefore, the main pulse is incident on a large volume spherical plasma close to the critical density.

Modeling the dynamics of a spherical droplet plasma would normally require three dimensional (3D) PIC simulations, which are still too expensive to realistically model the present experimental conditions. However, to gain useful insights into the differences between solid and droplet plasmas, we have carried out high-resolution 1D-PIC simulations with different density profiles that qualitatively mimic the expected density profiles from the droplet, at least in one dimension. An upper limit for the plasma scale length created by the prepulse can be obtained from the isothermal model. ${ }^{20,21}$ Assuming an absorbed flux of $2 \times 10^{13} \mathrm{~W} \mathrm{~cm}^{-2}$ for the prepulse, the plastic target would be initially heated to around $20 \mathrm{eV}$. One dimensional expansion at the sound speed would then give $L \sim c_{s} t \simeq 170 \mu \mathrm{m}$ after $10 \mathrm{~ns}$. Plasma cooling and geometrical factors will reduce this somewhat, but we can nevertheless expect density profiles with $L / \lambda$ $>10$. The droplets will expand even more due to their limited mass and the Mie enhancements in prepulse intensity.

The simulations were performed using BOPS, a $1 \mathrm{D} 2 \mathrm{~V}$ PIC code which exploits the Lorentz boost technique to handle oblique-incidence interactions. ${ }^{22,23}$ A series of runs was performed for various initial density scale lengths, keeping the total target mass (or charge) conserved, thus mimicking the expected droplet expansion. The inset of Fig. 3 shows the electron energy distribution for different scale lengths and the variation of hot electron numbers along with the hot electron temperature as a function of density scale length is shown in Fig. 3, which shows a fivefold increase in electrons above $20 \mathrm{keV}$ and an onset of "superhot" electrons $(>50 \mathrm{keV})$ as the profile is stretched from an abrupt step to an extended corona. Though the temperatures are a little smaller than those measured in the experiments, the qualitative trend is clearly visible. Perhaps the use of higher scale lengths and size limited features that are amenable from 3D simulations is mandatory to make a quantitative comparison. This series demonstrates that surprisingly high electron energies can be obtained even at modest intensities, provided the density profile is sufficiently extended, encouraging additional heating from parametric instabilities, as well as resonance absorption at near-normal incidence angles.

In summary, we have studied x-ray emission from intense laser irradiation of $15 \mu \mathrm{m}$ methanol droplets in comparison with that from solids. Our results show that the yields from droplet plasmas are larger by a factor of 68 at $2 \times 10^{15} \mathrm{~W} \mathrm{~cm}^{-2}$. A prepulse $10 \mathrm{~ns}$ ahead of the main pulse is essential for efficient hard $\mathrm{x}$-ray generation from the droplets. The preplasma from a spherical droplet is arguably more extensive than from the plane slab target and is conducive to efficient hot electron generation via resonance absorption. This idea is supported by 1D PIC simulations that mimic the long scale-length droplet profile.

${ }^{1}$ R. F. Service, Science 301, 154 (2003).

${ }^{2}$ B. A. Remington, D. Arnett, R. Paul, R. P. Drake, and H. Takabe, Science 284, 1488 (1999).

${ }^{3}$ T. Ditmire, J. Zweiback, V. P. Yanovsky, T. E. Cowan, G. Hays, and K. B. Wharton, Nature (London) 398, 489 (1999).

${ }^{4}$ T. Cowan, A. W. Hunt, T. W. Phillips, S. C. Wilks, M. D. Perry, C. Brown, W. Fountain, S. Hatchett, J. Johnson, M. H. Key, T. Parnell, D. M. Pennington, R. A. Snavely, and Y. Takahashi, Phys. Rev. Lett. 84, 903 (2000).

${ }^{5}$ S. Fritzler, V. Malka, G. Grillon, J. P. Rousseau, and F. Burgy, Appl. Phys. Lett. 83, 3039 (2003).

${ }^{6}$ M. Schnürer, Ch. Spielmann, P. Wobrauschek, C. Streli, N. H. Burnett, C. Kan, K. Ferencz, R. Koppitsch, Z. Cheng, T. Brabec, and F. Krausz, Phys. Rev. Lett. 80, 3236 (1998).

${ }^{7}$ P. P. Rajeev, P. Taneja, P. Ayyub, A. S. Sandhu, and G. Ravindra Kumar, Phys. Rev. Lett. 90, 115002 (2003).

${ }^{8}$ G. Kulcsar, D. AlMawlawi, F. W. Budnik, P. R. Herman, M. Moskovits, L. Zhao, and R. S. Marjoribanks, Phys. Rev. Lett. 84, 5149 (2000).

${ }^{9}$ V. P. Krainov and M. B. Smirnov, Phys. Rep. 370, 237 (2002).

${ }^{10}$ A. McPherson, B. D. Thompson, A. B. Borlsov, K. Boyer, and C. K. Rhodes, Nature (London) 370, 631 (1994).

${ }^{11}$ M. Schnürer, S. Ter-Avetisyan, S. Busch, E. Risse, M. P. Kalachnikov, W. Sandner and P. V. Nickles, J. Phys. B 23, 337 (2005), and references therein.

${ }^{12}$ L. Rymell and H. M. Hertz, Opt. Commun. 103, 105 (1993).

${ }^{13}$ R. Constantinescu, J. Jonkers, P. Hegemann, and M. Visser, Proc. SPIE 4146, 101 (2000).

${ }^{14}$ T. Donnelly, M. Rust, I. Weiner, M. Allen, R. A. Smith, C. A. Steinke, S. Wilks, J. Zweiback, T. E. Cowan, and T. Ditmire, J. Phys. B 34, L313 (2001).

${ }^{15}$ E. Parra, J. Fan, S. j. McNaught, and H. M. Milchberg, Appl. Phys. A: Mater. Sci. Process. 77, 317 (2003).

${ }^{16}$ H. C. Wu, W. Yu, T. J. Liang, X. Y. Peng, Z. Jin, Y. J. Li, Z. M. Sheng, X. W. Tang, and J. Zhang, Appl. Phys. B: Lasers Opt. 77, 687 (2003).

${ }^{17}$ M. Anand, C. P. Safvan, and M. Krishnamurthy, Appl. Phys. B: Lasers Opt. 81, 469 (2005).

${ }^{18}$ M. Anand, A. K. Dharmadhikari, J. A. Dharmadhikari, A. Mishra, D. Mathur, and M. Krishnamurthy, Chem. Phys. Lett. 372, 263 (2003).

${ }^{19}$ C. Favre, V. Boutou, S. C. Hill, W. Zimmer, M. Krenz, H. Lambrecht, J. Yu, R. K. Chang, L. Woeste, and J. P. Wolf, Phys. Rev. Lett. 89, 035002 (2002).

${ }^{20}$ C. E. Max and C. F. McKee, Phys. Rev. Lett. 39, 1336 (1977); for a more comprehensive treatement, see C. E. Max, in Laser Plasma Interaction, Les Houches 1980, edited by R. Balian and J. C. Adam (North-Holand, Amesterdam, 1982), Session XXXIV, p. 301; M. Rosen Proc. SPIE 1229, 160 (1990); W. L. Kruer, The Physics of Laser Plasma Intractions (Addison-Wesley, New York, 1988), and references therein.

${ }^{21}$ S. Bastiani, A. Rousse, J. P. Geindre, P. Audebert, C. Quoix, G. Hamoniaux, A. Antonetti, and J.-C. Gauthier, Phys. Rev. E 56, 7179 (1997).

${ }^{22}$ P. Gibbon and A. R. Bell, Phys. Rev. Lett. 68, 1535 (1992).

${ }^{23}$ P. Gibbon, A. A. Andreev, E. Lefebvre, G. Bonnaud, H. Ruhl, J. Delettrez, and A. Bell, Phys. Plasmas 6, 947 (1999) 